ARTICLE

\title{
Intratumoral immunotherapy using platelet-cloaked nanoparticles enhances antitumor immunity in solid tumors
}

Baharak Bahmani ${ }^{1,4}$, Hua Gong (10) 2,4, Brian T. Luk (1) 1,4, Kristofer J. Haushalter ${ }^{1}$, Ethel DeTeresa ${ }^{1}$, Mark Previti ${ }^{1}$,

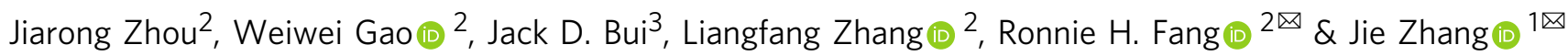

Intratumoral immunotherapy is an emerging modality for the treatment of solid tumors. Tolllike receptor (TLR) agonists have shown promise for eliciting immune responses, but systemic administration often results in the development of adverse side effects. Herein, we investigate whether localized delivery of the TLR agonist, resiquimod (R848), via platelet membrane-coated nanoparticles (PNP-R848) elicits antitumor responses. The membrane coating provides a means of enhancing interactions with the tumor microenvironment, thereby maximizing the activity of R848. Intratumoral administration of PNP-R848 strongly enhances local immune activation and leads to complete tumor regression in a colorectal tumor model, while providing protection against repeated tumor re-challenges. Moreover, treatment of an aggressive breast cancer model with intratumoral PNP-R848 delays tumor growth and inhibits lung metastasis. Our findings highlight the promise of locally delivering immunostimulatory payloads using biomimetic nanocarriers, which possess advantages such as enhanced biocompatibility and natural targeting affinities.

\footnotetext{
${ }^{1}$ Cello Therapeutics, Inc., San Diego, CA 92121, USA. ${ }^{2}$ Department of NanoEngineering, University of California San Diego, La Jolla, CA 92093, USA.

${ }^{3}$ Department of Pathology, University of California San Diego, La Jolla, CA 92093, USA. ${ }^{4}$ These authors contributed equally: Baharak Bahmani, Hua Gong,

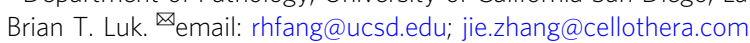


mmunotherapy has emerged as an effective therapeutic approach against cancer that harnesses the power of immune cells in the tumor microenvironment. Some recent approaches, including the use of immune checkpoint inhibitors against cytotoxic T-lymphocyte-associated protein 4 and programmed cell death protein 1 (Refs. ${ }^{1,2}$ ), as well as the adoptive transfer of chimeric antigen receptor (CAR) $\mathrm{T}$ cells ${ }^{3}$, have shown considerable promise. Despite the clinical success of such immunotherapies in the treatment of various cancer types ${ }^{4-7}$, each one still has its disadvantages that need to be overcome. For example, CAR $\mathrm{T}$ cell therapy has performed well against certain hematological cancers, but does not fare well against solid tumors ${ }^{8}$. Checkpoint blockade therapy is oftentimes associated with severe systemic side effects and only benefits a subset of patients with tumors that are in the correct immunological state ${ }^{9,10}$. One promising strategy to further expand the field of immunotherapy is the modulation of the tumor microenvironment via engagement of Toll-like receptors (TLRs) and inhibiting tumorpromoting immune signaling ${ }^{11-14}$. TLRs are mainly expressed by immune cells, and among them TLR7, an endosomal singlestranded RNA receptor, is predominantly expressed by macrophages, plasmacytoid dendritic cells, natural killer cells, and B cells ${ }^{15}$.

Resiquimod (R848), a small-molecule immunomodulator, belongs to the TLR7/8 agonist family. Upon binding of R848 to TLR7/8, multiple immunomodulatory cytokines, including interleukin 6 (IL-6), IL-12, and interferon a (IFNa) are released, therefore triggering a cascade of signaling pathways that leads to the activation of antigen-presenting cells (APCs) and polarization of $\mathrm{T}$ cell responses ${ }^{16-18}$. Despite extensive investigations into the role of TLRs in inducing innate immune responses to bacterial and viral pathogens, only recently has attention shifted to their role in anticancer immunosurveillance. TLR7/8 signaling can promote anticancer responses through activation of the central transcription factor nuclear factor $\kappa \mathrm{B}(\mathrm{NF}-\kappa \mathrm{B})^{19}$. It has been reported that TLR7/8 therapy leads to the expansion of tumor antigen-specific $\mathrm{CD}^{+} \mathrm{T}$ cells, which is important for the development of an effective antitumor immune response $\mathrm{e}^{20,21}$.

Although the systemic administration of R848 and other members of the TLR7 agonist family in combination with checkpoint inhibitors has proven advantageous in the treatment of squamous cell carcinoma, colon carcinoma, metastatic melanoma, and pancreatic cancer ${ }^{18,22-24}$, there are drawbacks limiting their clinical translation. For example, safety concerns were raised when multiple intravenous doses or oral administrations of smallmolecule TLR7 agonists caused adverse events such as fever, fatigue, headache, and hypertension in patients ${ }^{25-28}$. In addition, some reports suggested that systemic administration of R848 leads to rapid depletion of leukocytes and transient local immune insufficiency ${ }^{29}$. To overcome these challenges, intratumoral injection of TLR7 agonists has been investigated as a more clinically relevant route of administration to address solid tumors $^{30-34}$. The localization of immunostimulatory agents to the tumor microenvironment can convert it from a "cold" to a "hot" state, helping to kickstart antitumor immunity ${ }^{35}$. In order for intratumoral immunotherapies to be effective, it is necessary to confine the immune agonist payloads within the tumor site. However, the direct injection of free drug has the potential for systemic leakage that can lead to reduced efficacy, whereas targeted nanodelivery platforms are generally designed to be antigen-specific ${ }^{36}$, limiting their broad applicability.

Herein we report on the development of a platelet membranecloaked nanoparticle (PNP) specifically for the intratumoral delivery of R848 to treat solid tumors. The plasma membrane derived from human platelets, with its multitude of proteins, glycoproteins, and lipids, bestows platelet-mimicking properties such as selective adherence to cells in the tumor microenvironment ${ }^{37}$. Cell membrane coating is a facile approach for improving biocompatibility while enabling nanoparticle platforms to effectively interface with biological targets, such as tumors, through multimodal interactions ${ }^{38}$. We demonstrate that R848-loaded PNP (PNP-R848) exhibits prolonged retention at the tumor site and improves cellular interactions within the tumor microenvironment. This enables the nanoformulation to exert significant biological activity upon intratumoral administration, even at low R848 dosages that would otherwise be ineffective when administered systemically. In an MC38 murine colorectal adenocarcinoma model, it is shown that PNP-R848 promotes the strong activation of APCs within the draining lymph node (DLN) and increases immune infiltration. This ultimately leads to a potent antitumor response that facilitates the complete short-term rejection of established tumors while bestowing long-term immunity that protects against repeated and highly aggressive tumor re-challenges. The antitumor activity of the formulation is further confirmed in a metastatic 4T1 murine triple-negative breast cancer model.

\section{Results}

Nanoparticle synthesis and characterization. Given the multitude of interactions of platelets with other cell types and tissues $^{39-44}$, we aimed to leverage these unique abilities to design a nanoparticle platform incorporating natural targeting abilities. This was done by directly coating the membrane isolated from human platelets through a differential centrifugation and freeze-thaw process onto biocompatible and biodegradable polylactic acid (PLA) nanoparticle cores via sonication ${ }^{37}$, an energetically favorable process that promotes colloidal stabilization $^{45}$. The presence of phosphatidylserine, P-selectin, GPIba, and integrin $\alpha I I b \beta 3$ was confirmed on the surface of the platelet membrane ghosts by flow cytometry (Fig. 1a). Phosphatidylserine, P-selectin, and the full $\alpha \operatorname{IIb} \beta 3$ complex are expressed on the membrane surface upon platelet activation $^{46-49}$. GPIba is responsible for von Willebrand factormediated platelet adhesion ${ }^{50}$. P-selectin, $\alpha \mathrm{IIb} \beta 3$, and GPIba have all been implicated in cancer pathogenesis, suggesting important interactions with tumor cells ${ }^{51}$. Despite the activation state of the membrane, additional assays for thrombin and adenosine diphosphate (ADP) verified the successful physical removal of these intracellular platelet-activating molecules responsible for propagating thrombotic responses, thus mitigating safety concerns (Fig. 1b, c). Another detailed characterization of the platelet membrane has been previously reported ${ }^{37}$. Physicochemical characterizations revealed that membrane coating slightly increased the hydrodynamic size of both the bare PLA nanoparticle cores as well as the bare R848loaded nanoparticle cores (NP-R848) (Fig. 1d). Additionally, the surface zeta potential was similar between all samples (Fig. 1e). Transmission electron microscopy revealed that, compared to bare NP-R848, the final PNP-R848 formulation possessed a core-shell structure with a layer of membrane coating on the outside clearly separated from the nanoparticle core (Fig. 1f, g and Supplementary Fig. 1a). The nanoparticles remained stable in phosphate-buffered saline (PBS) over the course of 4 weeks, both when stored at room temperature and at $4{ }^{\circ} \mathrm{C}$ (Supplementary Fig. 1b). Quantification of drug loading revealed that $3.4 \mathrm{wt} \%$ of $\mathrm{R} 848$ could be encapsulated into the final formulation. Finally, the release of the R848 payload was studied over time, and the profiles for both the bare NP-R848 and coated PNP-R848 formulations matched closely, where more than $60 \%$ of the encapsulated payload was released within the first $24 \mathrm{~h}$ (Fig. 1h). 

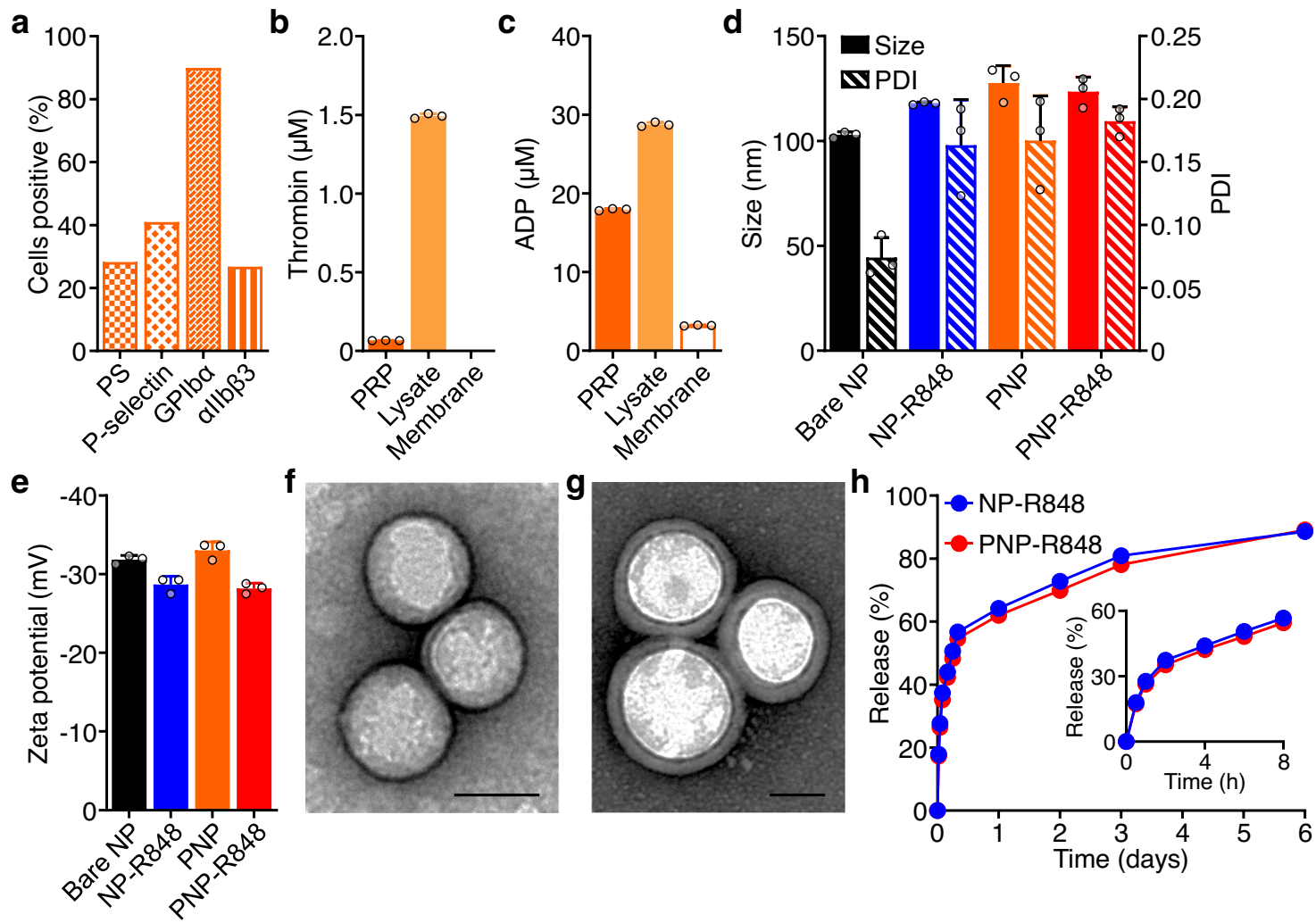

Fig. 1 Nanoparticle characterization. a Characterization of surface markers on platelet membrane, including phosphatidylserine (PS), P-selectin, GPIb $\alpha$, and $\alpha$ llb $\beta 3$. b, c Quantification of pro-thrombotic platelet-activating molecules thrombin (b) and adenosine diphosphate (ADP, c) in platelet-rich plasma $(\mathrm{PRP})$, platelet lysate, and purified platelet membrane $(n=3$, mean $+\mathrm{SD})$. d Average hydrodynamic diameter and polydispersity index (PDI) of bare nanoparticle (NP) cores, uncoated NP-R848, PNP, and PNP-R848 ( $n=3$, mean + SD). e Zeta potential of bare NP, NP-R848, PNP, and PNP-R848 ( $n=3$, mean + SD). $\mathbf{f}, \mathbf{g}$ Transmission electron microscopy visualization of uncoated NP-R848 (f) and coated PNP-R848 ( $\mathbf{g}$ ) with uranyl acetate negative staining (scale bars $=50 \mathrm{~nm}$; repeated 3 times). h Drug release profile from uncoated NP-R848 and coated PNP-R848 over 6 days (3 independent experiments). Source data are provided as a Source Data file.

Nanoparticle interaction with tumors. In order to assess the interaction of PNP with solid tumor cell types, we studied both binding and uptake in vitro. Fluorescent dye-labeled nanoparticles were incubated with a panel of murine and human cancer cells, including MC 38, HT-29, 4T1, and MDA-MB-231, at $4{ }^{\circ} \mathrm{C}$ for the binding study and $37^{\circ} \mathrm{C}$ for the uptake study. It was observed by flow cytometry that PNP much more readily bound to all four cancer cells compared with a polyethylene glycol (PEG)-coated nanoparticle (PEG-NP) control (Fig. 2a). These results correlated well with cellular uptake, which was also significantly higher for PNP than for PEG-NP in all of the cell lines (Fig. 2b). Considering the enhanced interaction of PNP with MC38 cells in vitro, we next tested the retention time of PNP in an MC38 tumor model in vivo. After allowing the tumors to establish, mice received a single intratumoral administration of dye-labeled PEG-NP or PNP, and the nanoparticles were tracked using a live imaging system over the course of 7 days (Fig. 2c, d). Initially, there was a similar drop in the amount of nanoparticles present within the tumor. As time progressed, the difference between the two groups increased, and the greatest contrast was observed at $48 \mathrm{~h}$, where on average $35 \%$ of the PNP remained, while only $11 \%$ of the PEG-NP was retained within the tumor. We then analyzed in vivo drug levels after intratumoral administration of R848-loaded nanoparticles. Compared with injection of free R848, which resulted in a large transient spike in serum R848 concentration, both PEG-NP-R848 and PNP-R848 were able to significantly limit systemic leakage (Fig. 2e). However, only PNP-R848, with its enhanced affinity to tumor cells, enabled prolonged drug persistence in the tumor tissue (Fig. 2f). Taken together, these studies demonstrated that the platelet membrane coating, which displays surface markers known to play a role in cancer cell binding ${ }^{52}$, was able to significantly increase nanoparticle affinity to the MC38 tumor cells compared with a more traditional PEG coating.

In vitro immunostimulatory activity. To directly assess the biological activity of the R848 payload, we incubated PNP-R848 with human reporter cell lines expressing either TLR7 or TLR8, which provide a colorimetric readout in response to NF- $\kappa B$ activation (Fig. 3a, b). The cells were incubated with free R848 or PNP-R848 for $21 \mathrm{~h}$, and our results showed that the activities of the two were roughly equivalent at the same drug concentration. As expected, PNP nanoparticles without drug loading showed minimal TLR7 and TLR8 activation. We next studied the biological effect of PNP-R848 on bone marrow-derived cells (BMDCs), and it was observed that the formulation could induce the upregulation of CD80 and CD86, two APC maturation markers that serve as co-stimulatory signals for mediating downstream immune responses (Fig. 3c, d). The expression levels of CD80 and CD86 were comparable to those induced by free R848, indicating that the loading of the payload into the nanoparticles did not affect their potent immunomodulatory activity. Additionally, we assessed the ability of PNP-R848 to elicit the production of proinflammatory cytokines such as IL-6, tumor necrosis factor a (TNFa), and IL-12 by BMDCs (Fig. 3e-g). After incubation with various concentrations of free R848 or PNP-R848, the culture supernatant was analyzed by enzyme-linked immunosorbent 

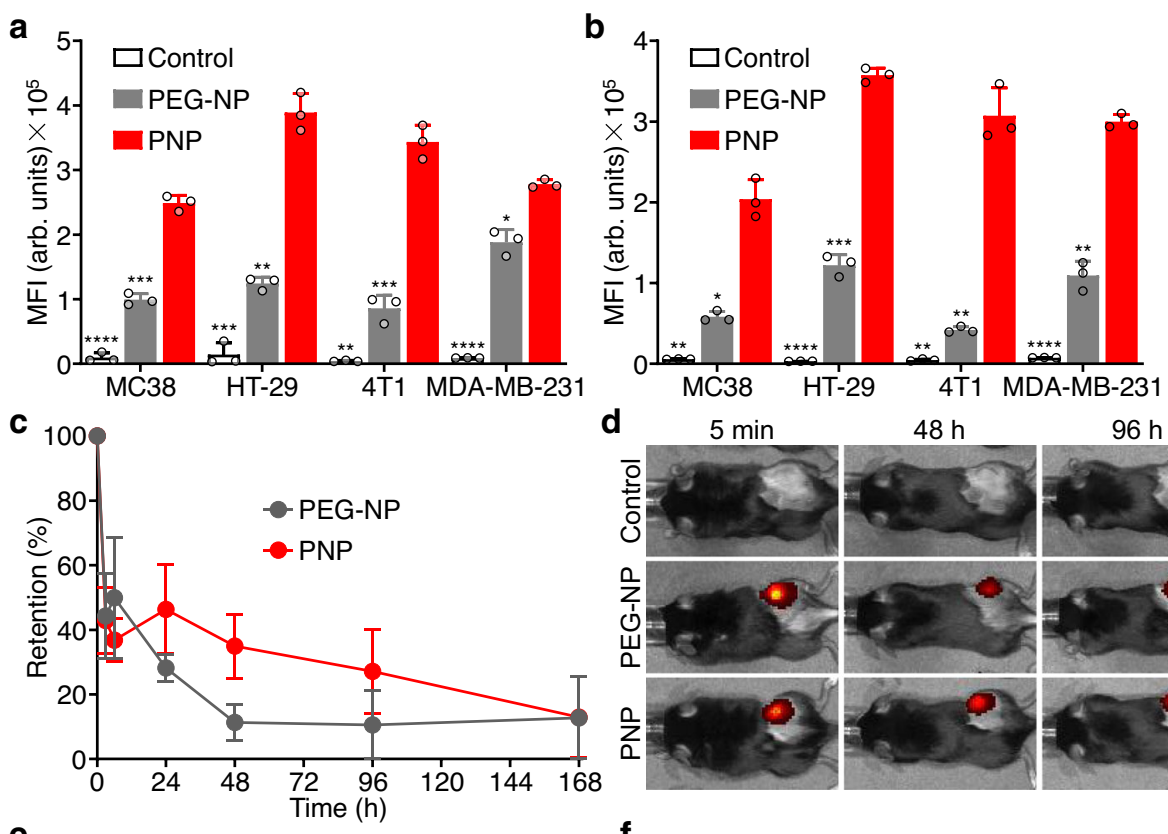

$96 \mathrm{~h}$
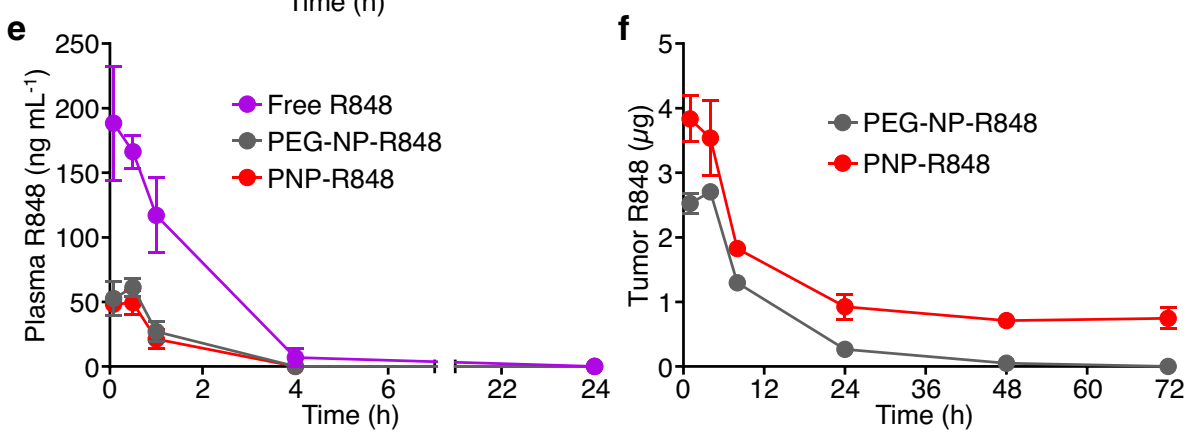

Fig. 2 Nanoparticle interaction with tumor cells. a, b Quantification of binding (a) and uptake (b) of PEG-NP and PNP by various cancer cells (MC38, HT29, 4T1, and MDA-MB-231) after incubation in vitro $\left(n=3\right.$, mean + SD; MFI = mean fluorescence intensity). ${ }^{\star} p<0.05,{ }^{\star \star} p<0.01,{ }^{\star \star \star} p<0.001,{ }^{\star \star \star *} p<$ 0.0001 (compared with PNP); two-way ANOVA. c Retention of PEG-NP or PNP over time after intratumoral administration into mice bearing MC38 tumors $(n=3$, mean \pm SEM). d Representative images from the study in (c) at $5 \mathrm{~min}, 48 \mathrm{~h}, 96 \mathrm{~h}$, and $168 \mathrm{~h}(\mathrm{H}=$ high fluorescent signal, $\mathrm{L}=$ low fluorescent signal). e Plasma levels of R848 after intratumoral administration of free R848, PEG-NP-R848, and PNP-R848 into mice bearing MC38 tumors ( $n=3$, mean \pm SEM). $\mathbf{f}$ Retention of R848 after intratumoral administration of PEG-NP-R848 and PNP-R848 into mice bearing MC38 tumors ( $n=3$, mean \pm SEM). Source data are provided as a Source Data file.

assays (ELISAs). For each cytokine that was studied, our results showed a dose-dependent release pattern that was similar for both samples. It was also confirmed that empty PNP, regardless of whether the platelet membrane was sourced from humans or mice, did not induce appreciable APC maturation or cytokine secretion (Supplementary Fig. 2); this supports the notion that the immune response elicited by PNP-R848 was driven largely by inclusion of the R848 payload.

Nanoparticle interaction with immune cells. We next sought to evaluate the interactions of the PNP formulation with various BMDC subpopulations (Fig. 3h, i). PNP showed a significant increase in both cell binding and uptake as compared to PEG-NP for all cell subtypes examined, including $\mathrm{CD}_{4} 5^{+}$leukocytes, $\mathrm{CD}_{11} \mathrm{~b}^{+}$macrophages, and $\mathrm{CD} 11 \mathrm{c}^{+}$dendritic cells. It is believed that the enhanced uptake of PNP by BMDCs may have contributed to the increased cytokine release observed in the preceding study. We next studied the in vivo interaction of the nanoformulation with tumor cell populations at various timepoints after intratumoral administration of dye-labeled PEG-NP and PNP (Fig. 3j-1). Overall, the uptake of PNP by the total population of cells in the tumor was significantly higher, evidenced by a significant increase in fluorescence intensity compared to PEG-NP. When immune cell subsets in the tumor were evaluated, higher uptake for PNP was also observed among CD45 ${ }^{+}$leukocytes and CD11c ${ }^{+}$dendritic cells across all timepoints.

Antitumor efficacy in a mouse colorectal cancer model. We evaluated the antitumor efficacy of PNP-R848 using an MC38 murine colon adenocarcinoma model in immunocompetent C57BL/6 mice (Fig. 4a). Each animal received a subcutaneous injection of $1 \times 10^{6} \mathrm{MC} 38$ cells in the right flank, and the average tumor size was allowed to reach $\sim 30-40 \mathrm{~mm}^{3}$. At this point, the mice started receiving one of the following treatments: $8 \%$ sucrose as a negative control, free R848, PEG-NP loaded with R848 (PEG-NP-R848), or PNP-R848, each at a drug dosage of $15 \mu \mathrm{g}$ per injection. Treatments were administered intratumorally every other day for a total of 3 times, after which the mice were monitored regularly to assess therapeutic efficacy (Fig. 4b-e). Rapid regression followed PNP-R848 treatment, and complete tumor eradication was observed for $100 \%$ of the mice. Tumor growth was considerably delayed when treating with either free R848 or PEG-NP-R848, but there was significant disease progression in a majority of the mice by 

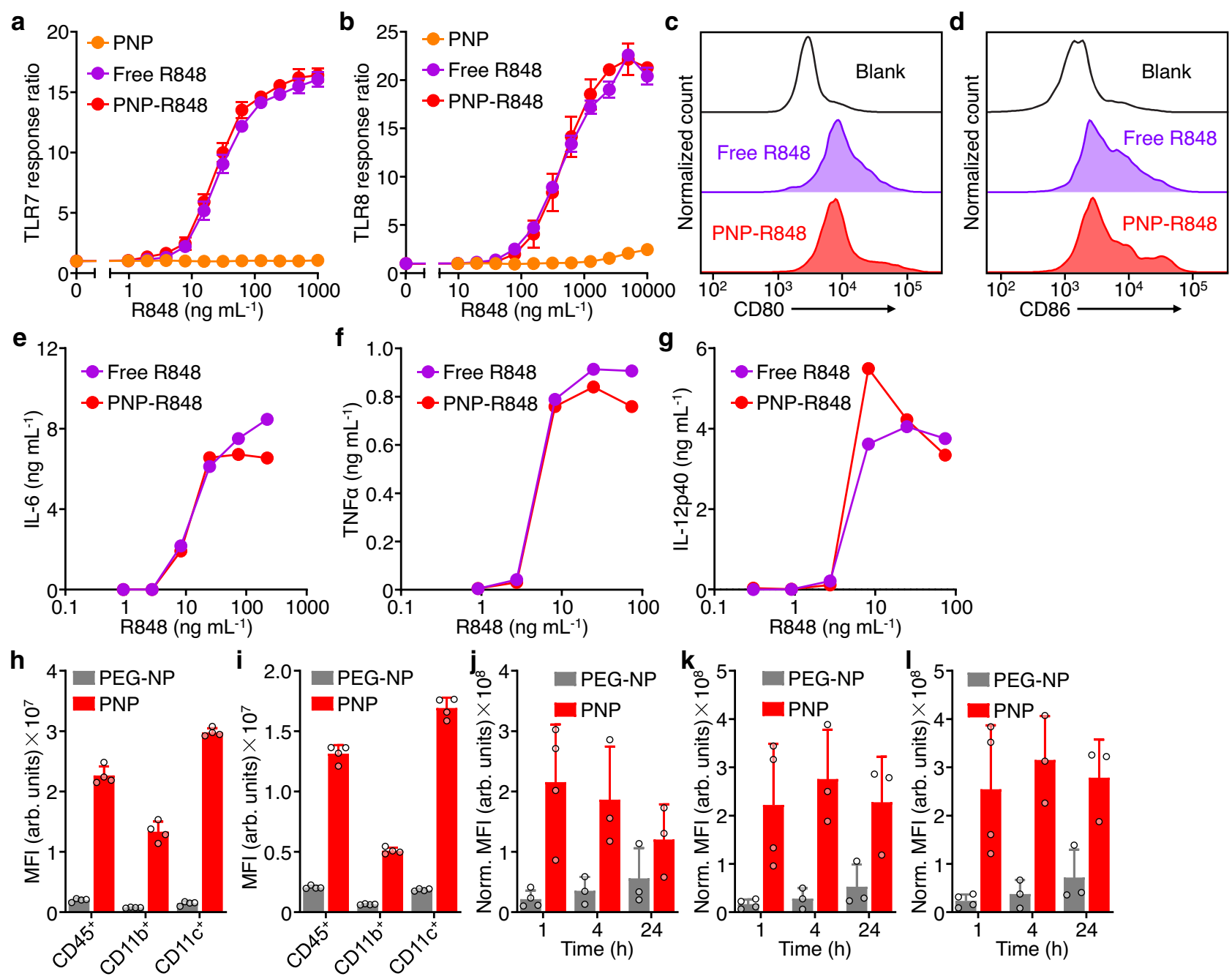

Fig. 3 Nanoparticle in vitro activity and interaction with immune cells. a, b Dose-dependent response of TLR7 (a) and TLR8 (b) reporter cell lines after incubation with PNP, free R848, and PNP-R848 ( $n=6$, mean + SD). c, d Expression of CD80 (c) and CD86 (d) by bone marrow-derived cells (BMDCs) after incubation with free R848 or PNP-R848. e-g Dose-dependent secretion of IL-6 (e), TNF $\alpha$ (f), and IL-12p40 (g) by BMDCs after incubation with free R848 and PNP-R848 ( $n=1$ for (e) and $n=2$ for ( $\mathbf{f}$ and $\mathbf{g})$ ). $\mathbf{h}, \mathbf{i}$ Quantification of binding (h) and uptake (i) of PEG-NP and PNP by immune cell subsets $\left(\mathrm{CD} 45^{+}, \mathrm{CD}_{11 \mathrm{~b}^{+}}\right.$, and $\left.\mathrm{CD} 11 \mathrm{c}^{+}\right)$after incubation with BMDCs in vitro $(n=4$, mean $+\mathrm{SD} ; \mathrm{MFI}=$ mean fluorescence intensity). $\mathbf{j}-\mathbf{I}$ In vivo uptake of PEG-NP and PNP by the total tumor cell population (j), CD45 ${ }^{+}$cells $(\mathbf{k})$, and CD11 $\mathrm{c}^{+}$cells $(\mathbf{I})$ at various timepoints after intratumoral administration $(n=4$ for $1 \mathrm{~h}$ timepoint and $n=3$ for rest of the timepoints, mean + SD). MFI was normalized based on the total cell number. Source data are provided as a Source Data file.

approximately 30 days after the start of treatment. In the end, both free R848 and PEG-NP-R848 treatments yielded a $28.6 \%$ long-term survival rate. We also evaluated treatment efficacy when reducing the drug dosage by 2.5 -fold to $6 \mu \mathrm{g}$ of R 848 per injection (Supplementary Fig. 3). In this case $87.5 \%$ of mice treated with PNP-R848 completely rejected the tumor challenge, and $28.6 \%$ of mice survived after treatment with PEGNP-R848. Interestingly, free R848 at the lower dosage outperformed the corresponding higher dosage treatment, with a $62.5 \%$ long-term survival rate. None of the treatments had a significant impact on the weight of the mice, suggesting that there was no acute toxicity. While there was a transient elevation of serum cytokine levels after treatment with PNP$\mathrm{R} 848$, the values all returned to baseline within $48 \mathrm{~h}$ (Supplementary Fig. 4). It should also be noted that both PEG-NP and PNP without any R848 loading had minimal effect on the progression-free survival of tumor-bearing mice, and any differences that were observed compared with the control group were statistically insignificant (Supplementary Fig. 5).
In order to determine if the surviving animals had developed long-term immunity against MC38 cancer cells, the mice were rechallenged with a 3-fold higher inoculum implanted subcutaneously into the right flank 56 days after initiation of the first treatment (Fig. 4c, d). For the survivors that had been treated with either dose of PNP-R848, the second tumor challenge was rejected at a $100 \%$ rate. Though animals treated with the $6-\mu \mathrm{g}$ dosage of free R848 initially demonstrated a $62.5 \%$ survival rate, the overall survival dropped to $37.5 \%$ after the tumor rechallenge, indicating inefficient development of an adaptive immune response against MC38 cells. The remainder of the surviving animals in the other groups all rejected the re-challenge, with no tumor progression observed at least 100 days after the start of the initial treatment. These results show that, while free R848 exhibits antitumor activity, it is not as efficient as the PNPR848 formulation at eliciting long-lasting immunity. Notably, mice that were treated with PNP-R848 all rejected a second rechallenge with a 5-fold higher dose of cancer cells performed 140 days after initial treatment (Fig. 4c, d). 

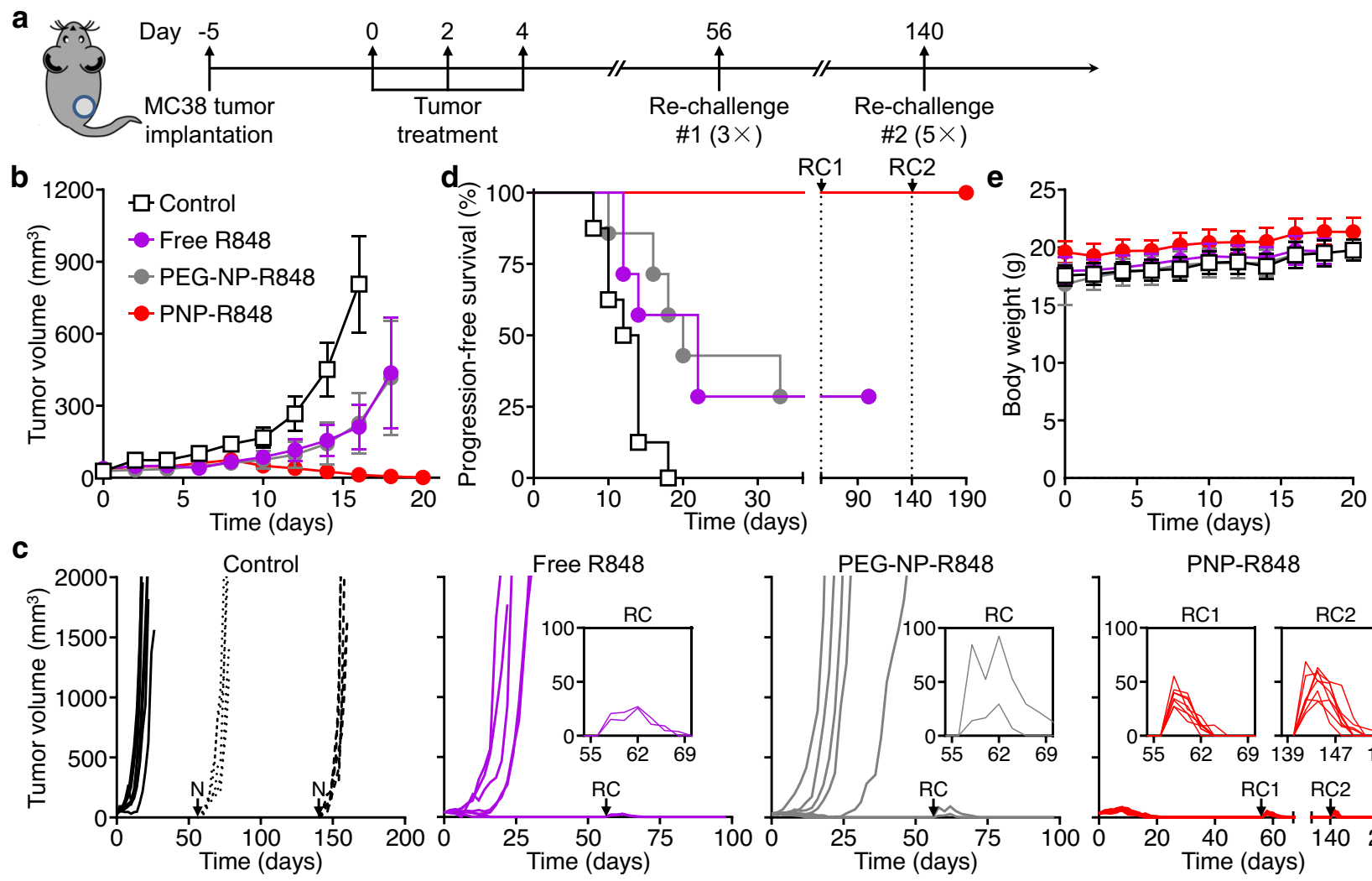

Fig. 4 Therapeutic antitumor efficacy in an MC38 tumor model. a Schematic timeline of the efficacy study. Tumors were treated with a sucrose control $(n=7)$, free R848 $(n=7)$, PEG-NP-R848 $(n=7)$, and PNP-R848 $(n=8)$. b Average tumor growth kinetics after treatment (mean \pm SEM). c Individual tumor growth kinetics after treatment $(\mathrm{N}=$ naive challenge, $\mathrm{RC}=$ re-challenge). The insets depict the growth kinetics after each re-challenge. $\mathbf{d}$ Progression-free survival (tumor size $<200 \mathrm{~mm}^{3}$ ) of mice after treatment. e Body weight of mice after treatment (mean $+\mathrm{SD}$ ). Source data are provided as a Source Data file.

We also assessed the therapeutic efficacy of PNP-R848 in combination with chemotherapy (Supplementary Fig. 6). While free doxorubicin administered intratumorally at a high dosage of $63 \mu \mathrm{g}$ prolonged the survival, the improvement was modest compared with PNP-R848 treatment. When combining the two treatment modalities, $100 \%$ of the mice survived the initial tumor challenge, although a greater than 10\% loss in body weight 6 days after the start of treatment suggested the presence of toxicity. Despite some promising initial results, all mice treated with doxorubicin, both with or without PNP-R848, succumbed after re-challenge with a 3 -fold higher dose of cancer cells. Given that leukodepletion is often a side effect of chemotherapy ${ }^{53}$, these results highlight the need for an intact immune response to achieve long-lasting antitumor protection.

Effect of treatment on immune cell populations in vivo. To elucidate the immune responses associated with treatment efficacy, the DLN from tumor-bearing mice were collected 7 days after administration of low-dose free R848 or PNP-R848 on the same schedule as above. We found that PNP-R848 was able to significantly elevate the expression of major histocompatibility complex II (MHC-II), a maturation marker, on $\mathrm{CD} 1 \mathrm{~b}^{+}$and $\mathrm{CD}_{11 \mathrm{c}^{+}}$APC subsets (Fig. 5a). No significant differences in MHC-II expression were observed in the same cell populations after free R848 treatment. Interestingly, the overall percentage of $\mathrm{CD}^{+} \mathrm{T}$ cells in the DLN on day 7 dropped in response to PNPR848 treatment (Fig. 5b), and this also held true for the proportion of $\mathrm{CD}^{+} \mathrm{T}$ cells (Fig. $5 \mathrm{c}$ ). Among the $\mathrm{T}$ cells that were present, the $\mathrm{CD} 4{ }^{+}$population had a significantly elevated proportion with the effector memory $\left(\mathrm{CD} 44^{\mathrm{hi}} \mathrm{CD} 62 \mathrm{~L}^{\text {low }}\right)$ and central memory (CD44hi CD62L ${ }^{\text {hi }}$ ) phenotypes (Fig. 5d). Since we observed a drop in the percentage of T cells in the DLN, we next assessed whether this was due to their migration into the tumor. The tumor tissue was histologically sectioned and stained for various immune cell subsets (Fig. 5e, f). Indeed, increased densities of both $\mathrm{CD}^{+}$and $\mathrm{CD} 8^{+} \mathrm{T}$ cells were found in the tumors of mice treated with PNP-R848 compared with free R848. Overall, our data indicate that PNP-R848, by improving tissue retention, was able to enhance the stimulation of APCs in the DLN, resulting in better priming of $\mathrm{T}$ cells and their subsequent recruitment into the tumor. This ultimately led to tumor eradication and the generation of memory $\mathrm{T}$ cells to fight subsequent tumor re-challenge.

Antitumor efficacy in a mouse breast cancer model. To further evaluate the applicability of PNP-R848 as a generalizable treatment against solid tumors, anticancer efficacy was tested in a syngeneic murine 4T1 triple-negative breast cancer model established using BALB/c mice (Fig. 6a). Each animal was subcutaneously implanted with $5 \times 10^{5}$ tumor cells in the right flank, and the average tumor size was allowed to reach $\sim 30-40 \mathrm{~mm}^{3}$ before treatment with either $8 \%$ sucrose, free R848, PEG-NPR848, or PNP-R848 at a drug dosage of $15 \mu \mathrm{g}$ per injection. The mice were treated every other day for a total of 5 times, and the tumor sizes and progression-free survival were monitored (Fig. 6b-d). Similar to the MC38 model, administration of PNPR848 resulted in significant inhibition of 4T1 tumor growth. With PNP-R848 treatment, progression-free survival was prolonged to 23 days, compared to 9 days for the control group. Both free R848 and PEG-NP-R848 exhibited an intermediate level of antitumor 

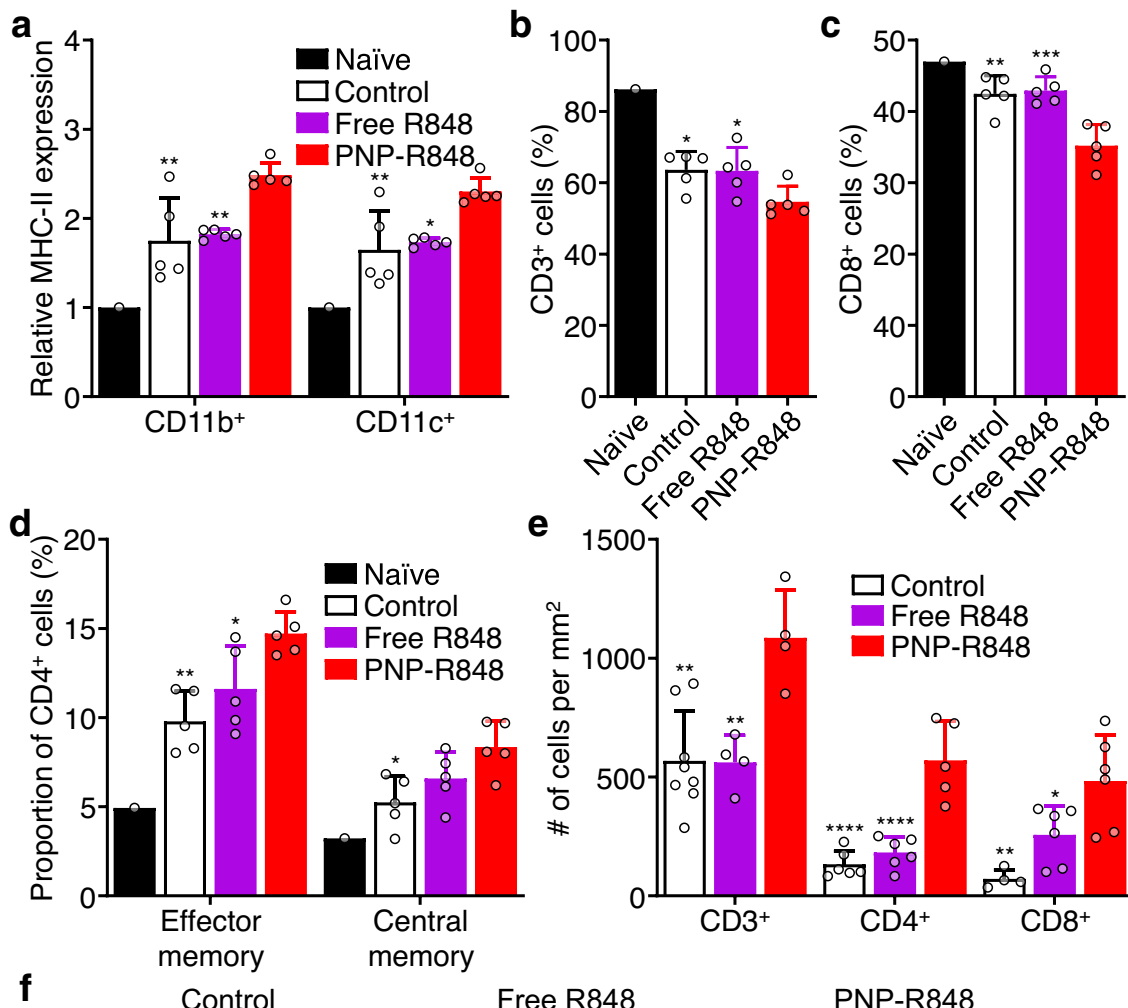

e

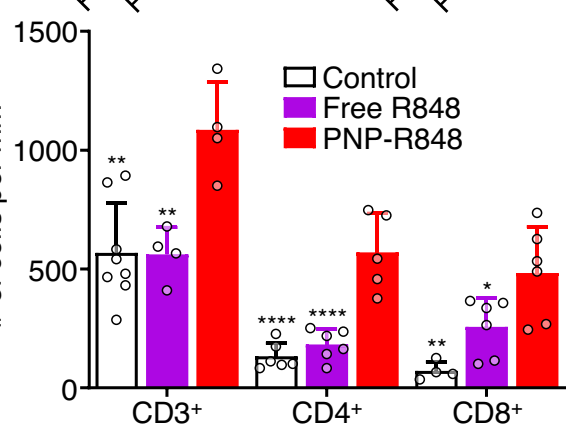

$\mathbf{f}$
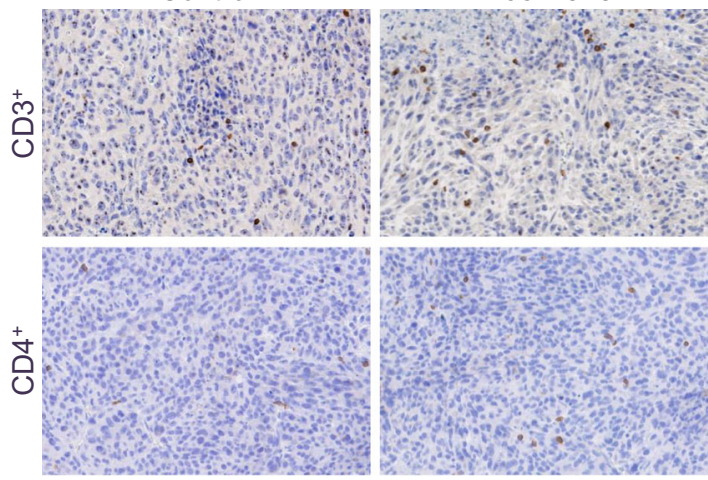

Free R848

PNP-R848
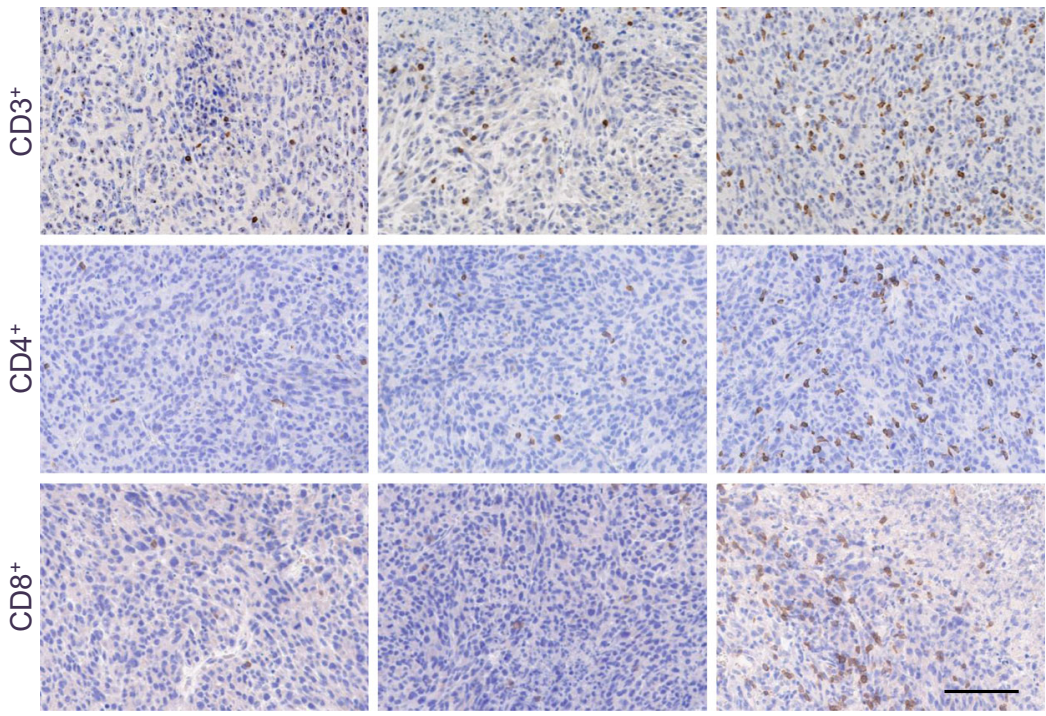

Fig. 5 Immune response to treatment in $\mathbf{M C 3 8}$ tumor-bearing mice. a Relative expression of MHC-II by CD11b ${ }^{+}$or CD11c ${ }^{+}$cells in the DLN from mice treated with free R848 and PNP-R848 $(n=5$, mean + SD). b Percentage of CD3+ cells within the CD45+ cell population of the DLN from mice treated with free R848 and PNP-R848 ( $n=5$, mean + SD). c Percentage of CD8 ${ }^{+}$cells within the CD3 ${ }^{+}$cell population of the DLN from mice treated with free R848 and PNP-R848 $\left(n=5\right.$, mean + SD). d Proportion of CD4 ${ }^{+}$T cells with the effector memory or central memory phenotypes in the DLN from mice treated with free R848 and PNP-R848 $\left(n=5\right.$, mean + SD). e Quantification of CD3 ${ }^{+}, C D_{4}+$, or CD8 ${ }^{+}$cell density in tumor sections from mice treated with free R848 and PNP-R848 ( $n=4$ for CD3+ Free R848, CD3+ PNP-R848, and CD8+ Control, $n=5$ for CD4+ PNP-R848, $n=6$ for CD4+ Control, CD4+ Free R848, CD8 ${ }^{+}$Free R848, and CD8 ${ }^{+}$PNP-R848, and $n=8$ for $C D 3^{+}$Control, mean + SD). $f$ Representative histological sections from the experiment in (e) (scale bar $=100 \mu \mathrm{m}$; brown = positive staining). All mice were treated on the same schedule as in Fig. 4 , and samples were collected on day 7 after the first treatment. ${ }^{\star} p<0.05,{ }^{\star \star} p<0.01,{ }^{\star \star \star} p<0.001,{ }^{\star \star \star \star} p<0.0001$ (compared with PNP-R848); one-way ANOVA. Source data are provided as a Source Data file.

efficacy. This trend was also reflected on day 30 after the first treatment, when the tumors were excised and weighed (Fig. 6e, f). Notably, PNP-R848 had a marked effect on the number of metastatic nodules in the lungs, reducing the average number per lung to 3 nodules from more than 50 for the control group (Fig. 6g).

\section{Discussion}

Here, we report on a biomimetic delivery vehicle to locally retain a potent immunomodulator at tumor sites. The TLR7 family of agonists stimulates dendritic cell activation and subsequent $\mathrm{T}$ cell priming, which leads to tumor-specific $\mathrm{T}$ cell immune responses and immunity $33,54,55$. There are some reports demonstrating that 

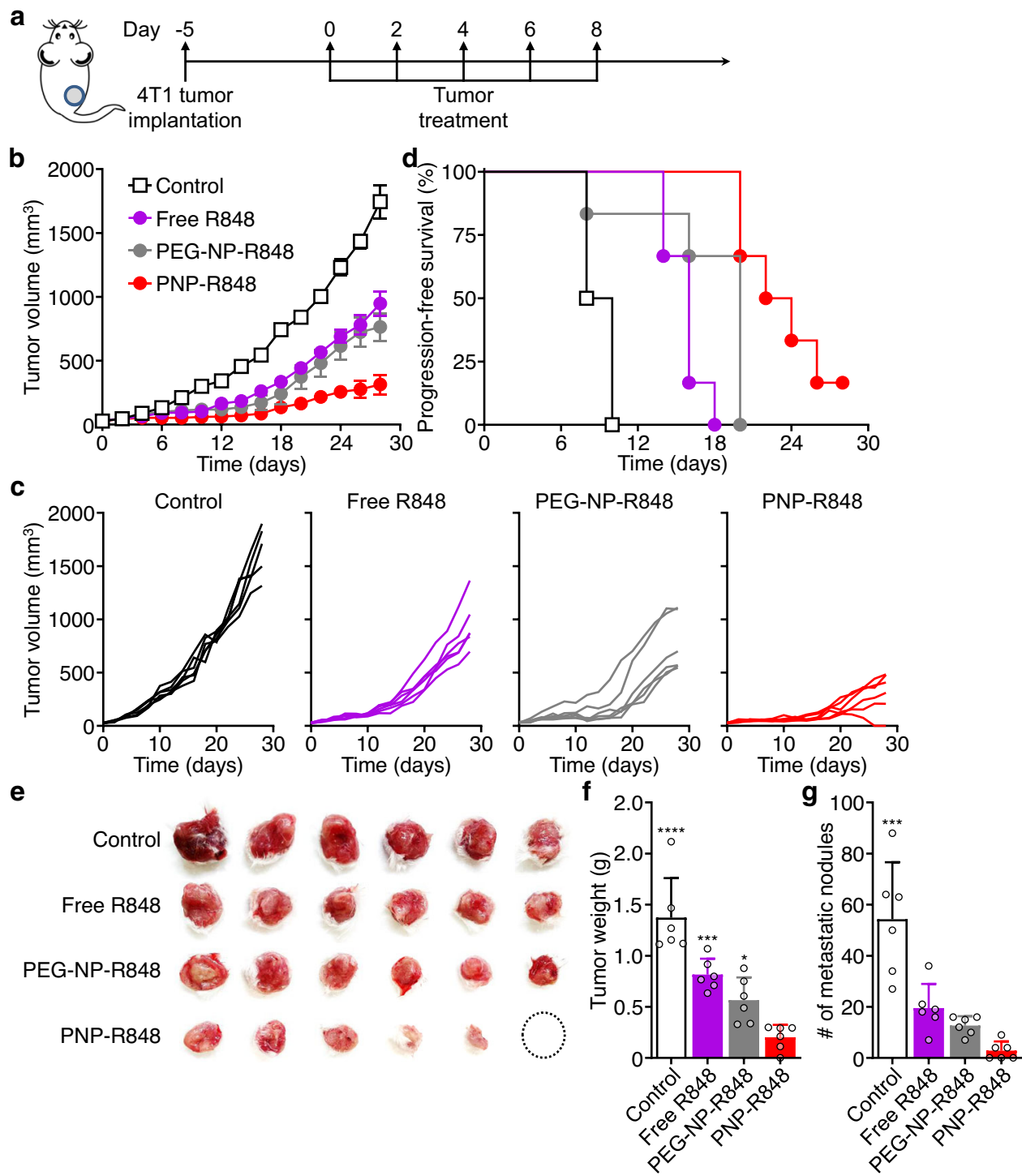

Fig. 6 Therapeutic antitumor efficacy in a 4T1 tumor model. a Schematic timeline of the efficacy study. Tumors were treated with free R848, PEG-NPR848, or PNP-R848 $(n=6)$. b Average tumor growth kinetics after treatment (mean \pm SEM). c Individual tumor growth kinetics after treatment. d Progression-free survival (tumor size $<200 \mathrm{~mm}^{3}$ ) of mice after treatment. e Images of tumors at day 30 post-treatment. $\mathbf{f}$ Average tumor weights at day 30 post-treatment (mean + SD). $\mathbf{g}$ Number of metastatic nodules in the lungs at day 30 post-treatment $\left(n=6,{ }^{2}\right.$ mean + SD). ${ }^{\star} p<0.05,{ }^{\star \star *} p<0.001$, ${ }^{* \star \star *} p<0.0001$ (compared with PNP-R848); one-way ANOVA. Source data are provided as a Source Data file.

systemic administration of R848 via the intravenous or intraperitoneal route promotes antitumor immune responses, but high drug dosages are generally required to achieve therapeutic efficacy ${ }^{18,56}$. In one case, mice bearing MC38 tumors were administered with a total of $600 \mu \mathrm{g}$ of R848, yet complete tumor regression was not observed ${ }^{56}$. In contrast, it has been shown that local delivery of R848 at more modest dosages, sometimes in combination with chemotherapy, can lead to complete tumor regression and long-term protective immunity 57,58 . It should be noted that considerable efficacy has generally been observed for intratumoral R848 only when combined with other immunostimulatory agents ${ }^{58,59}$. Our data suggest that the biomimetic platelet-derived membrane increased the interaction of PNPR848 with various cells in the tumor microenvironment, thereby enhancing the bioavailability of R848 at the tumor site and surrounding lymphoid tissue after local delivery. This enabled us to significantly reduce the required dose of R848 compared to previous studies while maintaining its therapeutic potential. Even at a low total dosage of $18 \mu \mathrm{g}$ per mouse, complete remission was observed in almost all mice receiving PNP-R848 alone in an MC38 colorectal tumor model.

We hypothesize that PNP-R848 administered intratumorally is able to affect tumor regression by triggering a local inflammatory response and activating the resident APCs, some of which can migrate to the DLN and promote a subsequent influx of primed $\mathrm{T}$ cells into the tumor tissue. We observed the infiltration of cytotoxic $\mathrm{CD}^{+}$cells into the tumor as early as 7 days posttreatment, which corresponded to the beginning of tumor size reduction in the efficacy study. Our data corroborate recent findings that intratumoral activation of TLR7/8 transforms the tumor microenvironment and induces immune cell infiltration into the tumor ${ }^{32}$. Additionally, the observed increase in effector and central memory T cells in the DLN supports the development of systemic adaptive antitumor immunity. When re-challenged with more aggressive tumor implantation protocols, the animals that had eradicated the initial MC38 tumors following intratumoral treatments with PNP-R848 exhibited strong immunity and completely rejected the new implants within 2 weeks. Notably, we 
also observed significant reduction in lung metastasis in a 4T1 breast cancer model after PNP-R848 treatment.

In conclusion, we have developed a biomimetic nanoformulation leveraging platelet membrane coating to enhance the delivery and retention of an immunostimulatory payload for intratumoral cancer immunotherapy. The membrane-coated nanoparticles efficiently interacted with cancer cells, leading to enhanced tumor retention in vivo and maximizing the activity of the encapsulated R848 payload. In an immunocompetent murine model of colorectal cancer, treatment with PNP-R848 was able to completely eradicate tumor growth, leading to long-term antitumor immunity that allowed all of the surviving mice to reject a subsequent re-challenge. The potent activity of the formulation was further corroborated in a murine model of triple-negative breast cancer, where significant reduction in metastasis was achieved. We believe that this approach for the localized delivery of small-molecule immunomodulators could be easily applied across a wide range of solid tumor types, providing a meaningful strategy for eliciting potent immune responses that could greatly enhance patient outcomes in the clinic.

\section{Methods}

Platelet membrane preparation and characterization. Human platelet-rich plasma (PRP) was purchased from the San Diego Blood Bank. The PRP was collected from voluntary donors by the Blood Bank following all relevant ethical regulations. No identifying information was associated with purchased units of PRP upon our receipt. To collect the platelet membrane, the PRP was first diluted $2 \times$ with a buffer consisting of $140 \mathrm{mM} \mathrm{NaCl}$ (Fisher Chemical), $2.7 \mathrm{mM} \mathrm{KCl}$ (Fisher Chemical), $3.8 \mathrm{mM}$ 4-(2-hydroxyethyl)-1-piperazineethanesulfonic acid (HEPES; Acros), $5 \mathrm{mM}$ ethylene glycol-bis( $\beta$-aminoethyl ether)- $N, N, N^{\prime}, N^{\prime}$-tetraacetic acid (Bioworld), and $2 \mu \mathrm{M}$ prostaglandin E1 (PGE1; AdooQ BioScience), followed by centrifugation at $2000 \mathrm{~g}$ for $15 \mathrm{~min}$ with no brake. The supernatant was removed and the platelets were resuspended with a lysis buffer containing a

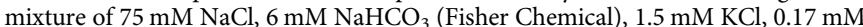
$\mathrm{Na}_{2} \mathrm{HPO}_{4}$ (Fisher Chemical), $0.5 \mathrm{mM} \mathrm{MgCl}$ (Alfa Aesar), $20 \mathrm{mM}$ HEPES, $1 \mathrm{mM}$ ethylenediaminetetraacetic acid (Fisher Chemical), $1 \mu \mathrm{M}$ PGE1, 0.01\%

NP40 surfactant (Boston Bioproducts), and protease inhibitors (Thermo Scientific). The platelet membrane was derived by a repeated freeze-thaw process. The platelet mixture was frozen at $-80^{\circ} \mathrm{C}$, thawed at room temperature, and pelleted by centrifugation at $21,100 \mathrm{~g}$ for $10 \mathrm{~min}$. The pellet was then resuspended in the lysis buffer, and the freeze-thaw was repeated two more times. After the repeated washes, the membrane was suspended in water for coating onto the nanoparticle cores.

Quantification of total membrane protein concentration was performed using a Pierce BCA protein assay kit (Life Technologies). Flow cytometry was used to probe for the expression of specific surface markers on the platelet membrane using FITC-conjugated annexin V (Biolegend), Alexa488-conjugated anti-human Pselectin (AK4; Biolegend), Alexa647-conjugated anti-human GPIba (HIP1; Biolegend), and Alexa647-conjugated anti-human aIIb $\beta 3$ (PAC-1; Biolegend). The probes (1:200 dilution) were incubated with purified platelet membrane in PBS (Gibco) for $30 \mathrm{~min}$ in the dark at room temperature. After incubation, the membrane was washed by centrifugation at $21,100 \mathrm{~g}$. Data were collected using a Becton Dickinson Accuri C6 flow cytometer equipped with BD Accuri C6 Plus software and analyzed with FlowJo V10 software.

\section{Nanoparticle synthesis and physicochemical characterization. R848-loaded} nanoparticles were synthesized using a single emulsion process. First, PLA (R202H; Evonik) and R848 (BOC Sciences) were dissolved in an organic phase consisting of benzyl alcohol (Acros) and ethyl acetate (Fisher Chemical) at concentrations of 60 and $10 \mathrm{mg} \mathrm{mL}^{-1}$, respectively. The mixture was then added to $5 \times$ volume of icecold outer phase media consisting of $10 \mathrm{mM}$ Tris $\mathrm{pH} 7.5$ (Invitrogen) with $0.2 \mathrm{wt} \%$ sodium cholate (Alfa Aesar) and 7 vol\% ethyl acetate. This solution was homogenized at 12,000 rpm for $90 \mathrm{~s}$ using a Kinematica Polytron PT 3100 homogenizer before being passed through a Microfluidics LM20 Microfluidizer (outfitted with a $\mathrm{Y}$ chamber) 3 times. This mixture was then added to an equal volume of outer phase media, and the solvent was evaporated overnight in a fume hood while stirring at $200 \mathrm{rpm}$. Unloaded nanoparticle cores were fabricated using the same procedure without R848 in the organic phase. Platelet membrane coating was performed by sonication of the R848-loaded or unloaded nanoparticle cores with platelet membrane at a polymer-to-platelet membrane protein mass ratio of 1:0.7 for $2 \mathrm{~min}$ in a Branson CPX3800H ultrasonic bath at a frequency of $40 \mathrm{kHz}$. This process is expected to lyse any intact platelet vesicles present in the membrane preparation. PEG-coated nanoparticles were fabricated using the same procedure as for the nanoparticle cores, but using PEG-conjugated PLA (PolySciTech) to replace $10 \mathrm{wt} \%$ of the unconjugated PLA. To prepare nanoparticles loaded with 1,1'-dioctadecyl-3,3,3',3'-tetramethylindodicarbocyanine (DiD; Biotium), the dye was added to a $10 \mathrm{mg} \mathrm{mL}^{-1}$ PLA solution in acetone at $0.1 \mathrm{wt} \%$ of the polymer. Then, $2 \mathrm{~mL}$ of this solution was added dropwise to $4 \mathrm{~mL}$ of water to form the dyeloaded nanoparticle cores. After overnight solvent evaporation, the nanoparticles were coated with platelet membrane by sonication. Hydrodynamic nanoparticle size and surface zeta potential were measured by dynamic light scattering using a Malvern Zetasizer Nano ZS. For imaging, the nanoparticles were stained with 0.2 wt\% uranyl acetate (Electron Microscopy Sciences) and visualized with an FEI Tecnai Spirit G2 BioTWIN transmission electron microscope. For stability characterization, uncoated NP-R848 or coated PNP-R848 were suspended at $1 \mathrm{mg} \mathrm{mL}$ ${ }^{-1}$ in PBS and stored either at room temperature or at $4^{\circ} \mathrm{C}$. At weekly intervals, the size of the samples was measured by dynamic light scattering using a Malvern Zetasizer Nano ZS. All nanoformulations were passed through a $0.2-\mu \mathrm{m}$ filter for sterilization prior to in vitro and in vivo experiments.

Quantification of platelet activation factors. PRP, platelet lysate, and purified platelet membrane were prepared and examined for the platelet-activating molecules thrombin and ADP using a SensoLyte 520 thrombin activity assay kit (Anaspec) and a PicoProbe ADP assay kit (BioVision), respectively, based on the manufacturers' instructions. To prepare the PRP, lysate, and platelet membrane samples, $1 \mathrm{~mL}$ of PRP was first diluted $3 \times$ and divided into 3 groups. The first group was used directly, the second group was washed and underwent one freezethaw cycle to produce the platelet lysate, and the third group was processed to completion into purified membrane. All samples were resuspended to the same volume with PBS prior to performing the assays.

Drug loading and release. R848 loading was analyzed using a reverse-phase ultrahigh-performance liquid chromatography (UHPLC) method. The UHPLC system consisted of a binary gradient pump, in-line degasser, autosampler and Thermo Scientific Vanquish photodiode array detector. Separation and quantitative analysis of R848 were achieved on a $3.5 \mu \mathrm{m}$ Waters XBridge ${ }^{\mathrm{mm}} \mathrm{C} 18$ column $(2.1 \times 150 \mathrm{~mm})$ with the mobile phase flowing at a rate of $1.0 \mathrm{~mL} \mathrm{~min}{ }^{-1}$ and a detection wavelength of $227 \mathrm{~nm}$. Mobile phase A consisted of $10 \mathrm{mM}$ sodium phosphate (Fisher Chemical) with $0.1 \%$ triethylamine (Acros) and $\mathrm{pH}$ adjusted to 2.45 , while mobile phase B consisted of $100 \%$ acetonitrile (Fisher Chemical). The acquisition run time for each analysis was $6.5 \mathrm{~min}$ with a gradient consisting of $15 \%$ mobile phase B from 0 to $3 \mathrm{~min}, 45 \%$ mobile phase $B$ from 3 to $5 \mathrm{~min}$, and $15 \%$ mobile phase $B$ from 5.1 to $6.5 \mathrm{~min}$. The samples were first diluted in acetonitrile and then diluted in a combination of 30\% acetonitrile and 70\% $0.1 \mathrm{~N}$ hydrochloric acid (Acros). They were then injected into the column after a series of six standard injections prepared by diluting R848 in 100\% acetonitrile. Drug release kinetics from PNPR848 were evaluated utilizing $20 \mathrm{kDa}$ dialysis cassettes (Thermo Scientific).

Reconstituted samples were transferred to the cassettes via a syringe with a 21gauge needle and dialyzed against a large volume of PBS. The dissolution experiments were run at room temperature while stirring the PBS solution at $280 \mathrm{rpm}$ for 6 days. Samples were collected at various timepoints $(30 \mathrm{~min}, 1 \mathrm{~h}, 2 \mathrm{~h}, 4 \mathrm{~h}, 6 \mathrm{~h}, 8 \mathrm{~h}$, 1 day, 2 days, 3 days, and 6 days) and analyzed by UHPLC.

In vitro binding and uptake of PNP by murine and human cancer cells. MC38 murine colon adenocarcinoma cells (Kerafast) and MDA-MB-231 human mammary gland adenocarcinoma cells (HTB-26; American Type Culture Collection) were cultured in Dulbecco's modified Eagle's medium (Gibco) supplemented with $10 \%$ fetal bovine serum (Corning). 4T1 murine mammary gland cancer cells (CRL2539; American Type Culture Collection) were cultured in RPMI-1640 medium (Gibco) supplemented with 10\% fetal bovine serum. HT-29 human colorectal adenocarcinoma cells (HTB-38; American Type Culture Collection) were cultured in McCoy's 5a medium (Gibco) supplemented with $10 \%$ fetal bovine serum. For the binding study, DiD-loaded PNP or PEG-NP were incubated with $5 \times 10^{5}$ MC38, HT-29, 4T1, or MDA-MB-231 cells in $100 \mu \mathrm{L}$ of media. The final nanoparticle concentration for this incubation was $0.2 \mathrm{mg} \mathrm{mL}^{-1}$. The incubation was performed for $30 \mathrm{~min}$ at $4{ }^{\circ} \mathrm{C}$ in order to minimize endocytic uptake, after which the cells were washed 3 times with PBS and examined using flow cytometry. For the uptake study, the incubation was instead performed for 10 min at $37^{\circ} \mathrm{C}$. Data were collected using a Becton Dickinson Accuri C6 flow cytometer equipped with BD Accuri C6 Plus software and analyzed with FlowJo V10 software.

TLR activation assays. HEK-Blue hTLR7 and HEK-Blue hTLR8 reporter cells (Invivogen) were cultured as directed by the manufacturer. For the dose-response experiments, $20 \mu \mathrm{L}$ of PNP, free R848, or PNP-R848 were loaded into 96-well cell culture plates at $10 \times$ the desired final concentration $\left(0.977\right.$ to $\left.1000 \mathrm{ng} \mathrm{mL}^{-1}\right)$. The cultured reporter cells were rinsed with warm PBS, resuspended in $1 \mathrm{~mL}$ warm PBS, and then detached from culture flasks by gentle scraping. The cells were diluted to a concentration of $2.2 \times 10^{5}$ cells $\mathrm{mL}^{-1}$ in HEK-Blue detection medium (Invivogen), whereupon $180 \mu \mathrm{L}$ of the cell suspension was immediately added to the sample dilutions. Absorbance at $655 \mathrm{~nm}$ was measured after $21 \mathrm{~h}$ of incubation at $37^{\circ} \mathrm{C}$ in $5 \% \mathrm{CO}_{2}$. 
Nanoparticle activity on BMDCs. All animal experiments were performed in accordance with guidelines approved by the Institutional Animal Care and Use Committee of the University of California San Diego. Animals were maintained in standard housing at $68-75^{\circ} \mathrm{F}, 40-60 \%$ relative humidity, and $12 \mathrm{~h}$ light/dark cycles. Female C57BL/6 mice (Charles River Laboratories) were euthanized via $\mathrm{CO}_{2}$ asphyxiation. Intact tibias were isolated from each mouse, dipped briefly into $70 \%$ ethanol, and stored in RPMI cell culture media (Gibco) over ice. Both ends of each tibia were cut and each bone was flushed with $10 \mathrm{~mL}$ of RPMI using a syringe attached with a 23-gauge needle. Bone marrow cells were collected and washed by centrifugation at $320 \mathrm{~g}$ for $9 \mathrm{~min}$. Finally, cells were passed through a $50-\mu \mathrm{m}$ cell strainer (Corning). For cytokine release and co-stimulatory marker characterization, BMDC cells were counted, and $5 \times 10^{5}$ cells were plated per well in 6 -well plates. Various concentrations of free R848 and PNP-R848 were added to the cells and incubated at $37^{\circ} \mathrm{C}$ for $24 \mathrm{~h}$. Afterwards, the supernatant was assayed using ELISA kits for IL-6 (BD Biosciences), TNF $\alpha$ (R\&D Systems), and IL-12p40 (R\&D Systems). The cells were washed and scraped from the plates, followed by staining with FITC-conjugated anti-mouse CD45 (30-F11; BD Biosciences; 1:400 dilution), PE-conjugated anti-mouse CD80 (16-10A1; BD Biosciences; 1:400 dilution), and APC-conjugated anti-mouse CD86 (GL-1; Biolegend; 1:400 dilution). Data were collected using a Becton Dickinson Accuri C6 flow cytometer equipped with BD Accuri C6 Plus software and analyzed with FlowJo V10 software (Supplementary Fig. 7). In a separate experiment, empty PNP fabricated from mouse platelets (mPNP) or human platelets (hPNP), free R848, or PNP-R848 were added to the cells at an R848 concentration of $24.6 \mathrm{ng} \mathrm{mL}^{-1}$ (or equivalent nanoparticle amount) and incubated at $37^{\circ} \mathrm{C}$ for $24 \mathrm{~h}$. Lipopolysaccharide (LPS; Invivogen) at $20 \mathrm{ng} \mathrm{mL}^{-1}$ was used as a positive control. Afterwards, the supernatant and cells were analyzed as described above.

BMDC binding and uptake. For cell binding studies, DiD-loaded PNP or PEG-NP were incubated with $1 \times 10^{6} \mathrm{BMDC}$ cells in $100 \mu \mathrm{L}$ media at a final nanoparticle concentration of $0.2 \mathrm{mg} \mathrm{mL}^{-1}$. Incubation was performed for $30 \mathrm{~min}$ at $4^{\circ} \mathrm{C}$, after which the cells were washed 3 times with PBS and subsequently stained using FITC-conjugated anti-mouse CD45 (30-F11; BD Biosciences; 1:400 dilution), PEconjugated anti-mouse CD11b (M1/70; Biolegend; 1:400 dilution), and PE/Cy7conjugated anti-mouse CD11c (N418; Biolegend; 1:400 dilution). For uptake studies, incubation was performed for $10 \mathrm{~min}$ at $37^{\circ} \mathrm{C}$. Data were collected using a Becton Dickinson Accuri C6 flow cytometer equipped with BD Accuri C6 Plus software and analyzed with FlowJo V10 software (Supplementary Fig. 7).

In vivo interaction with tumors. All animal experiments were performed in accordance with ethical regulations for animal testing and research and were approved by the Institutional Animal Care and Use Committee of the University of California San Diego. To develop tumors, $1 \times 10^{6}$ MC38 cells were implanted subcutaneously into the right flank of 6 -week old female C57BL/6 mice. Tumor volumes were calculated using the equation: volume $=\left(\right.$ length $\times$ width $\left.^{2}\right) / 2$. For the tumor retention study, the average tumor size was allowed to reach $100 \mathrm{~mm}^{3}$, after which the mice were administered with $8 \%$ sucrose as the negative control $(n=3)$, DiD-labeled PNP $(n=3)$, and DiD-labeled PEG-NP $(n=3)$. Each animal received one intratumoral injection and was imaged using a Xenogen IVIS 200 system at various timepoints, including $5 \mathrm{~min}, 3 \mathrm{~h}, 6 \mathrm{~h}, 24 \mathrm{~h}, 48 \mathrm{~h}, 96 \mathrm{~h}$, and $168 \mathrm{~h}$ with the same acquisition time and filter settings. Acquired images were analyzed by the Xenogen Living Image software 3.0 to quantify the fluorescence intensity of the tumors and to determine tumor retention percentage.

To assess interaction with immune cells, mice with tumors with an average volume of $100 \mathrm{~mm}^{3}$ were intratumorally administered with DiD-labeled PNP or PEG-NP. At $1 \mathrm{~h}, 4 \mathrm{~h}$, and $24 \mathrm{~h}$, groups of mice were euthanized, and the tumor tissue was processed into single-cell suspensions by digesting in a solution containing collagenase IV (Sigma-Aldrich) and DNase type IV (Sigma-Aldrich) at final concentrations of $1 \mathrm{mg} \mathrm{mL}^{-1}$ and $10 \mu \mathrm{g} \mathrm{mL}^{-1}$, respectively. The cells were stained using FITC-conjugated anti-mouse CD45 (1:400 dilution), PE/Cy7conjugated anti-mouse CD11c (1:400 dilution), and LIVE/DEAD Fixable Aqua Stain (Invitrogen). Data were collected using a Becton Dickinson Accuri C6 flow cytometer equipped with BD Accuri C6 Plus software and analyzed with FlowJo V10 software (Supplementary Fig. 7).

In vivo quantification of R848. For serum analysis, $1 \times 10^{6}$ MC38 cells were implanted subcutaneously into the right flank of 6-week old female C57BL/6 mice. When the average tumor size reached $100 \mathrm{~mm}^{3}$, mice were randomized into three groups and received a single intratumoral treatment of $8 \%$ sucrose $(n=3)$, PEGNP-R848 $(n=3)$, and PNP-R848 $(n=3)$. Each group received $15 \mu \mathrm{g}$ of R848. Blood samples were collected in BD Microtainer collection tubes at $5 \mathrm{~min}, 30 \mathrm{~min}$, $1 \mathrm{~h}, 4 \mathrm{~h}, 24 \mathrm{~h}, 48 \mathrm{~h}$, and $72 \mathrm{~h}$ post-treatment. The blood samples were centrifuged at $15,000 \mathrm{~g}$ for $2 \mathrm{~min}$ to separate the plasma, which was then analyzed using UHPLC for levels of R848. To assess R848 tumor retention, $1 \times 10^{6}$ MC38 cells were implanted by subcutaneous injection into the right and left flank of each C57BL/6 mouse. When the average tumor size reached $100 \mathrm{~mm}^{3}$, mice were randomized into two groups and received a single intratumoral treatment of PEG-NP-R848 ( $n$ $=12)$ and PNP-R848 $(n=12)$ into each tumor. At $1 \mathrm{~h}, 4 \mathrm{~h}, 8 \mathrm{~h}, 24 \mathrm{~h}, 48 \mathrm{~h}$, and $72 \mathrm{~h}$ post-treatment, two mice from each group were euthanized, and their tumors were collected and processed for analysis of R848 levels by UHPLC.

Therapeutic efficacy in a murine MC38 tumor model. Mice were implanted with $1 \times 10^{6}$ MC38 cells subcutaneously into the right flank, which were allowed to grow to an average size of $\sim 30-40 \mathrm{~mm}^{3}$. The mice were then intratumorally treated every other day for a total of 3 times. The treatment groups included: $8 \%$ sucrose, free R848, PEG-NP-R848, and PNP-R848. Each group received 6 or $15 \mu$ g of R848 per treatment. The injection volume was $30 \mu \mathrm{L}$ for all the treatments, and delivery was done via syringe with a 31-gauge needle. Care was taken to administer the solution slowly to prevent leakage. Tumor growth and mouse weight were monitored every other day. Progression-free survival was defined as tumor volume $<200 \mathrm{~mm}^{3}$, a threshold above which, based on our experience with the model, complete tumor regression is unlikely. All the mice that rejected the initial MC38 inoculum were rechallenged subcutaneously using $3 \times 10^{6}$ MC38 cells on day 56 after the start of the first treatment. Mice in the PNP-R848 treatment group that were tumor-free at the end of the initial re-challenge study were re-challenged a second time with $5 \times 10^{6}$ MC38 cells on day 140 . For each re-challenge, 5 naive C57BL/6 mice that received the same MC38 tumor cell challenge were used as controls to verify tumorigenicity.

Systemic cytokine release. Mice were implanted with $1 \times 10^{6}$ MC 38 cells subcutaneously into the right flank, which were allowed to grow to an average size of $150-200 \mathrm{~mm}^{3}$. The mice received intratumoral injections of $8 \%$ sucrose and PNPR848. Blood samples were collected in BD Microtainer collection tubes at $2 \mathrm{~h}, 8 \mathrm{~h}$, $24 \mathrm{~h}$, and $48 \mathrm{~h}$ post-treatment. The blood samples were centrifuged at $15,000 \mathrm{~g}$ for 2 min to separate the plasma, which was then analyzed using ELISA kits for IL-6, TNFa, and IL-12p40.

Therapeutic efficacy with unloaded nanocarriers. Mice were implanted with $1 \times$ $10^{6}$ MC38 cells subcutaneously into the right flank, which were allowed to grow to an average size of $\sim 30-40 \mathrm{~mm}^{3}$. The mice received intratumoral treatments every other day for a total of 4 times. The treatment groups included: $8 \%$ sucrose, PEG$\mathrm{NP}$, and PNP. The nanoparticles used in this study were empty and did not contain R848. Tumor growth and mouse weight were monitored every other day. Progression-free survival was defined as tumor volume $<200 \mathrm{~mm}^{3}$.

Therapeutic efficacy in combination with doxorubicin. Mice were implanted with $1 \times 10^{6}$ MC38 cells subcutaneously into the right flank, which were allowed to grow to an average size of $\sim 30-40 \mathrm{~mm}^{3}$. The mice received intratumoral treatments every other day for a total of 3 times. The treatment groups included: $8 \%$ sucrose free doxorubicin, and doxorubicin + PNP-R848. Mice received $63 \mu \mathrm{g}$ of doxorubicin and $15 \mu \mathrm{g}$ of R848 per dose. For the combination treatment, doxorubicin and PNP-R848 were mixed pre-administration and the animal received one intratumoral injection containing both. Tumor growth and mouse weight were monitored every other day. Progression-free survival was defined as tumor volume $<200 \mathrm{~mm}^{3}$. All the mice that rejected the initial MC38 inoculum were re-challenged subcutaneously using $3 \times 10^{6} \mathrm{MC} 38$ cells on day 56 after the start of the first treatment. Five naive C57BL/6 mice that received the same MC38 tumor cell challenge were used as controls to verify tumorigenicity.

In vivo immune profiling. Mice bearing MC38 tumors were treated on the same schedule as the antitumor efficacy study with $8 \%$ sucrose, free R848, and PNPR848. Each group received $6 \mu \mathrm{g}$ of R848. The mice were then euthanized 7 days after the first treatment, and the inguinal DLN (on the same side as the tumor) was processed into a single-cell suspension by shearing the tissue using a $50-\mu \mathrm{m}$ cell strainer. Cells were stained with Fixable Viability Stain 450 (BD Biosciences) and different antibodies, including BV510-conjugated anti-mouse CD3 (17A2; Biolegend; 1:400 dilution), FITC-conjugated anti-mouse CD4 (RM4-5; eBiosciences; 1:400 dilution), APC/Cy7-conjugated anti-mouse CD8 (53-6.7; Invitrogen; 1:400 dilution), PerCP/Cy5.5-conjugated anti-mouse CD62L (MEL-14; eBioscience; 1:400 dilution), APC-conjugated anti-mouse CD44 (IM7; BD Biosciences; 1:400 dilution), V500-conjugated anti-mouse CD45 (30-F11; BD Biosciences; 1:400 dilution), APC-conjugated anti-mouse MHC-II (M5/114.15.2; Tonbo Bioscience; 1:400 dilution), APC/Cy7-conjugated anti-mouse CD11b (M1/70; BD Biosciences 1:400 dilution), and PE/Cy7-conjugated anti-mouse CD11c (1:400 dilution). Data were collected using a Becton Dickinson FACSCanto II flow cytometer equipped with BD FACSDiva software and analyzed with FlowJo V10 software (Supplementary Fig. 7). Tumor tissues were fixed in formalin (Fisher Scientific) for $24 \mathrm{~h}$ and were then transferred into $70 \%$ ethanol prior to histological sectioning by the Moores Cancer Center Tissue Technology Shared Resource. Tumor sections were stained for mouse $\mathrm{CD} 3, \mathrm{CD} 4$, and $\mathrm{CD} 8$ using AEC substrate and counterstained with Mayer's Hematoxylin. Slides were imaged with a Hamamatsu Nanozoomer 2.0HT slide scanner and post-acquisition quantifications were made using QuPath v0.2.0 (open-source software from Github).

Therapeutic efficacy in a murine 4T1 tumor model. Female BALB/c mice (Charles River Laboratories) were implanted with $5 \times 10^{5} 4 \mathrm{~T} 1$ cells subcutaneously into the right flank, which were allowed to grow to an average size of $\sim 30-40 \mathrm{~mm}^{3}$ 
The mice were then treated every other day for a total of 5 treatments. The treatment groups included: $8 \%$ sucrose $(n=6)$, free R848 $(n=6)$, PEG-NP-R848 $(n=6)$, and PNP-R848 $(n=6)$. Each group received $15 \mu \mathrm{g}$ of R848 per treatment. The injection volume was $30 \mu \mathrm{L}$ for all the treatments, and delivery was done via syringe with a 31-gauge needle. Tumor growth and mouse weight were monitored every other day. Progression-free survival was defined as tumor volume $<200 \mathrm{~mm}^{3}$. The study was terminated 30 days after the first treatment, and the tumors and lungs were harvested. For metastatic nodule counting, lung tissues were fixed using Bouin's solution (Electron Microscopy Sciences).

Graph generation and statistical analysis. GraphPad Prism 8 software was used for all graph generation and statistical analysis.

Reporting summary. Further information on research design is available in the Nature Research Reporting Summary linked to this article.

\section{Data availability}

All data are available within the Article, Supplementary Information or available from the corresponding authors upon reasonable request. Source data are provided with this paper.

Received: 25 May 2020; Accepted: 5 March 2021; Published online: 31 March 2021

\section{References}

1. Tumeh, P. C. et al. PD-1 blockade induces responses by inhibiting adaptive immune resistance. Nature 515, 568-571 (2014).

2. Vilain, R. E. et al. Dynamic changes in PD-L1 expression and immune infiltrates early during treatment predict response to PD-1 blockade in melanoma. Clin. Cancer Res. 23, 5024-5033 (2017).

3. Sharma, P., Wagner, K., Wolchok, J. D. \& Allison, J. P. Novel cancer immunotherapy agents with survival benefit: recent successes and next steps. Nat. Rev. Cancer 11, 805-812 (2011).

4. Mihara, K. et al. Activated T-cell-mediated immunotherapy with a chimeric receptor against CD38 in B-cell non-Hodgkin lymphoma. J. Immunother. 32, 737-743 (2009).

5. Buchbinder, E. \& Hodi, F. S. Cytotoxic T lymphocyte antigen-4 and immune checkpoint blockade. J. Clin. Invest. 125, 3377-3383 (2015).

6. Chen, L. \& Han, X. Anti-PD-1/PD-L1 therapy of human cancer: past, present, and future. J. Clin. Invest. 125, 3384-3391 (2015).

7. Grupp, S. A. et al. Chimeric antigen receptor-modified T cells for acute lymphoid leukemia. N. Engl. J. Med. 368, 1509-1518 (2013).

8. Newick, K., O'Brien, S., Moon, E. \& Albelda, S. M. CAR T cell therapy for solid tumors. Annu. Rev. Med. 68, 139-152 (2017).

9. Michot, J. M. et al. Immune-related adverse events with immune checkpoint blockade: a comprehensive review. Eur. J. Cancer 54, 139-148 (2016)

10. Kluger, H. M. et al. PD-L1 studies across tumor types, its differential expression and predictive value in patients treated with immune checkpoint inhibitors. Clin. Cancer Res. 23, 4270-4279 (2017).

11. Liu, C., Han, C. \& Liu, J. The role of Toll-like receptors in oncotherapy. Oncol. Res. 27, 965-978 (2019).

12. Matijevic, T. \& Pavelic, J. Toll-like receptors: Cost or benefit for cancer? Curr. Pharm. Des. 16, 1081-1090 (2010).

13. Medzhitov, R. Toll-like receptors and innate immunity. Nat. Rev. Immunol. 1, 135-145 (2001).

14. Braunstein, M. J., Kucharczyk, J. \& Adams, S. Targeting Toll-like receptors for cancer therapy. Target. Oncol. 13, 583-598 (2018).

15. Lester, S. N. \& Li, K. Toll-like receptors in antiviral innate immunity. J. Mol. Biol. 426, 1246-1264 (2014).

16. Vasilakos, J. P. \& Tomai, M. A. The use of Toll-like receptor $7 / 8$ agonists as vaccine adjuvants. Expert Rev. Vaccines 12, 809-819 (2013).

17. Aranda, F. et al. Trial watch: Toll-like receptor agonists in oncological indications. Oncoimmunology 3, e29179 (2014)

18. Michaelis, K. A. et al. The TLR7/8 agonist R848 remodels tumor and host responses to promote survival in pancreatic cancer. Nat. Commun. 10, 4682-4682 (2019).

19. Schön, M. P. \& Schön, M. TLR7 and TLR8 as targets in cancer therapy. Oncogene 27, 190-199 (2008).

20. Dovedi, S. J. et al. Systemic delivery of a TLR7 agonist in combination with radiation primes durable antitumor immune responses in mouse models of lymphoma. Blood 121, 251-259 (2013).

21. Cheadle, E. J. et al. A TLR7 agonist enhances the antitumor efficacy of obinutuzumab in murine lymphoma models via NK cells and CD4 T cells. Leukemia 31, 1611-1621 (2017).
22. Nishii, N. et al. Systemic administration of a TLR7 agonist attenuates regulatory $\mathrm{T}$ cells by dendritic cell modification and overcomes resistance to PD-L1 blockade therapy. Oncotarget 9, 13301-13312 (2018).

23. Rodell, C. B. et al. TLR7/8-agonist-loaded nanoparticles promote the polarization of tumour-associated macrophages to enhance cancer immunotherapy. Nat. Biomed. Eng. 2, 578-588 (2018).

24. Dummer, R. et al. An exploratory study of systemic administration of the Toll-like receptor-7 agonist $852 \mathrm{~A}$ in patients with refractory metastatic melanoma. Clin. Cancer Res. 14, 856-864 (2008).

25. Harrison, L., Astry, C., Kumar, S. \& Yunis, C. Pharmacokinetics of 852A, an imidazoquinoline Toll-like receptor 7-specific agonist, following intravenous, subcutaneous, and oral administrations in humans. J. Clin. Pharmacol. 47, 962-969 (2007).

26. Pockros, P. J. et al. Oral resiquimod in chronic HCV infection: safety and efficacy in 2 placebo-controlled, double-blind phase IIa studies. J. Hepatol. 47, 174-182 (2007).

27. Savage, P. et al. A phase I clinical trial of imiquimod, an oral interferon inducer, administered daily. Br. J. Cancer 74, 1482-1486 (1996).

28. Goldstein, D. et al. Administration of imiquimod, an interferon inducer, in asymptomatic human immunodeficiency virus-infected persons to determine safety and biologic response modification. J. Infect. Dis. 178, 858-861 (1998).

29. Gunzer, M. et al. Systemic administration of a TLR7 ligand leads to transient immune incompetence due to peripheral-blood leukocyte depletion. Blood 106, 2424-2432 (2005).

30. Currie, A. J. et al. Targeting the effector site with IFN- $\alpha \beta$-inducing TLR ligands reactivates tumor-resident CD8 $\mathrm{T}$ cell responses to eradicate established solid tumors. J. Immunol. 180, 1535-1544 (2008).

31. Schmidt, C. Immune system's Toll-like receptors have good opportunity for cancer treatment. J. Natl Cancer Inst. 98, 574-575 (2006).

32. Mullins, S. R. et al. Intratumoral immunotherapy with TLR7/8 agonist MEDI9197 modulates the tumor microenvironment leading to enhanced activity when combined with other immunotherapies. J. Immunother. Cancer 7, 244-244 (2019)

33. Singh, M. et al. Effective innate and adaptive antimelanoma immunity through localized TLR7/8 activation. J. Immunol. 193, 4722-4731 (2014).

34. Narayanan, J. S. S. et al. Irreversible electroporation combined with checkpoint blockade and TLR7 stimulation induces antitumor immunity in a murine pancreatic cancer model. Cancer Immunol. Res. 7, 1714-1726 (2019).

35. Trujillo, J. A., Sweis, R. F., Bao, R. \& Luke, J. J. T cell-inflamed versus non-T cell-inflamed tumors: a conceptual framework for cancer immunotherapy drug development and combination therapy selection. Cancer Immunol. Res. 6, 990-1000 (2018).

36. Dehaini, D., Fang, R. H. \& Zhang, L. Biomimetic strategies for targeted nanoparticle delivery. Bioeng. Transl. Med. 1, 30-46 (2016).

37. $\mathrm{Hu}, \mathrm{C}$. M. et al. Nanoparticle biointerfacing by platelet membrane cloaking. Nature 526, 118-121 (2015).

38. Fang, R. H., Kroll, A. V., Gao, W. \& Zhang, L. Cell membrane coating nanotechnology. Adv. Mater. 30, 1706759 (2018).

39. Born, G. V. \& Cross, M. J. The aggregation of blood platelets. J. Physiol. 168, 178-195 (1963)

40. Fitzgerald, J. R., Foster, T. J. \& Cox, D. The interaction of bacterial pathogens with platelets. Nat. Rev. Microbiol. 4, 445-457 (2006).

41. Haemmerle, M., Stone, R. L., Menter, D. G., Afshar-Kharghan, V. \& Sood, A. K The platelet lifeline to cancer: challenges and opportunities. Cancer Cell 33, 965-983 (2018)

42. Kieffer, N. \& Phillips, D. R. Platelet membrane glycoproteins: Functions in cellular interactions. Annu. Rev. Cell Biol. 6, 329-357 (1990).

43. McEver, R. P. The clinical significance of platelet membrane glycoproteins. Hematol. Oncol. Clin. North Am. 4, 87-105 (1990).

44. Xu, X. R., Yousef, G. M. \& Ni, H. Cancer and platelet crosstalk: opportunities and challenges for aspirin and other antiplatelet agents. Blood 131, 1777-1789 (2018).

45. Luk, B. T. et al. Interfacial interactions between natural RBC membranes and synthetic polymeric nanoparticles. Nanoscale 6, 2730-2737 (2014).

46. Csongradi, E. et al. Increased levels of platelet activation markers are positively associated with carotid wall thickness and other atherosclerotic risk factors in obese patients. Thromb. Haemost. 106, 683-692 (2011).

47. Kannan, M., Ahmad, F. \& Saxena, R. Platelet activation markers in evaluation of thrombotic risk factors in various clinical settings. Blood Rev. 37, 100583 (2019).

48. Saleh, H. M., Attia, E. A., Onsy, A. M., Saad, A. A. \& Abd Ellah, M. M. Platelet activation: a link between psoriasis per se and subclinical atherosclerosis - a case-control study. Br. J. Dermatol. 169, 68-75 (2013).

49. Durrant, T. N., van den Bosch, M. T. \& Hers, I. Integrin $\alpha \operatorname{IIb} \beta 3$ outside-in signaling. Blood 130, 1607-1619 (2017).

50. Resendiz, J. C., Feng, S., Ji, G. \& Kroll, M. H. von Willebrand factor binding to platelet glycoprotein Ib-IX-V stimulates the assembly of an $\alpha$-actinin-based signaling complex. J. Thromb. Haemost. 2, 161-169 (2004). 
51. Gay, L. J. \& Felding-Habermann, B. Contribution of platelets to tumour metastasis. Nat. Rev. Cancer 11, 123-134 (2011).

52. Stone, J. P. \& Wagner, D. D. P-selectin mediates adhesion of platelets to neuroblastoma and small cell lung cancer. J. Clin. Invest. 92, 804-813 (1993).

53. Bally, M. B., Nayar, R., Masin, D., Cullis, P. R. \& Mayer, L. D. Studies on the myelosuppressive activity of doxorubicin entrapped in liposomes. Cancer Chemother. Pharmacol. 27, 13-19 (1990).

54. Chi, H. et al. Anti-tumor activity of Toll-like receptor 7 agonists. Front. Pharmacol. 8, 304 (2017)

55. Drobits, B. et al. Imiquimod clears tumors in mice independent of adaptive immunity by converting pDCs into tumor-killing effector cells. J. Clin. Invest. 122, 575-585 (2012).

56. Schmid, D. et al. T cell-targeting nanoparticles focus delivery of immunotherapy to improve antitumor immunity. Nat. Commun. 8, 1747 (2017).

57. Stathopoulos, A. et al. Development of immune memory to glial brain tumors after tumor regression induced by immunotherapeutic Toll-like receptor $7 / 8$ activation. Oncoimmunology 1, 298-305 (2012).

58. Nie, Y. et al. Development of a curative therapeutic vaccine (TheraVac) for the treatment of large established tumors. Sci. Rep. 7, 14186 (2017).

59. Da Silva, C. G. et al. Co-delivery of immunomodulators in biodegradable nanoparticles improves therapeutic efficacy of cancer vaccines. Biomaterials 220, 119417 (2019).

\section{Acknowledgements}

We thank Alborz Amirsadeghi and Mao Yin for their help in the early development stage.

\section{Author contributions}

B.B., H.G., R.H.F., and J. Zhang conceived and designed the experiments. B.B., H.G., B.T L., K.J.H., E.D., M.P., J. Zhou, and W.G. performed the experiments. The manuscript was written by B.B., R.H.F., and J. Zhang. J.D.B. and L.Z. helped with study design and critically revised the manuscript. All authors discussed the results and reviewed the manuscript.

\section{Competing interests}

These authors declare the following competing financial interests: B.B., B.T.L., K.J.H., E D., M.P., and J. Zhang are employees of Cello Therapeutics. L.Z. has financial interest in Cello Therapeutics. The remaining authors declare no competing interest.

\section{Additional information}

Supplementary information The online version contains supplementary material available at https://doi.org/10.1038/s41467-021-22311-z.

Correspondence and requests for materials should be addressed to R.H.F. or J.Z.

Peer review information Nature Communications thanks the anonymous reviewers for their contribution to the peer review of this work. Peer review reports are available.

Reprints and permission information is available at http://www.nature.com/reprints

Publisher's note Springer Nature remains neutral with regard to jurisdictional claims in published maps and institutional affiliations.

cc (i) Open Access This article is licensed under a Creative Commons Attribution 4.0 International License, which permits use, sharing, adaptation, distribution and reproduction in any medium or format, as long as you give appropriate credit to the original author(s) and the source, provide a link to the Creative Commons license, and indicate if changes were made. The images or other third party material in this article are included in the article's Creative Commons license, unless indicated otherwise in a credit line to the material. If material is not included in the article's Creative Commons license and your intended use is not permitted by statutory regulation or exceeds the permitted use, you will need to obtain permission directly from the copyright holder. To view a copy of this license, visit http://creativecommons.org/ licenses/by/4.0/.

(C) The Author(s) 2021 